Proceedings of the Thirteenth International Conference on Climbing and Walking Robots and the Support Technologies for Mobile Machines, CLAWAR 2010, in press.

\title{
Experimental Evaluation of a Goniometer For the Identification of Anatomical Joint Motions
}

\author{
V. A. D. CAI* , B. BRU, P. BIDAUD, \\ V. HAYWARD and V. PASQUI \\ Institut des Systèmes Intelligents et de Robotique, Université Pierre et Marie Curie, \\ Paris, 75005, France \\ *E-mail: cai,bru,bidaud,hayward,pasqui@isir.upmc.fr \\ www.isir.upmc.fr

\section{F. GOSSELIN}

CEA, LIST, Laboratoire de Robotique Interactive, Fontenay Aux Roses, 92265, France E-mail: florian.gosselin@cea.fr

\begin{abstract}
This paper exposes the experimental evaluation of a new technique for the estimation of the instantaneous helical axis of movement of human anatomical joints. The measurement technique, using a six degrees of freedom spatial electro-goniometer, is tested onto a simple revolute joint and onto a subject's knee. A motion capture system with active optical markers is used at the same time in order to validate the measurement results.
\end{abstract}

Keywords: Instantaneous Screw Axis (ISA), Knee Joint Motion.

\section{Motivation}

The notion of screw motion (Chasle's theorem, 1830), which relates to the description of the instantaneous motion of a body in space in terms of a simultaneous translation and rotation around a single axis, is particularly useful concept in biomechanics since anatomical joints never behave like true lower pairs, but invariably undergo composite motions through their range. Hence, there has long been an interest in the determination of the instantaneous screw axis of the relative motion between two segments of a limb. ${ }^{5}$ In the knee, for instance, general six degree-of-freedom spatial motions must be considered.

The International Society of Biomechanics (ISB) recommends the use of the Helical Axis (HA) method. ${ }^{9}$ Various studies are aimed at evaluating the reliability of the estimation of the instantaneous screw axis (ISA) based 
Proceedings of the Thirteenth International Conference on Climbing and Walking Robots and the Support Technologies for Mobile Machines, CLAWAR 2010, in press.

on measurement acquired from anatomical limbs..$^{3,6,8}$ It is found a common problem is a lack of reliability of the estimate at low angular velocities and restrictive assumptions as to the nature of the motion.

Here, we propose to determine the instantaneous screw axis as well as the corresponding translational and angular velocities. The methods apply both to data acquired from optical motion capture systems or by mechanical goniometers. While optical tracking $\vec{z}$ have been applied relatively recently to the estimation of these quaptities, the mechanical goniometer has a long history. ${ }^{7}$ Recently, we developed such goniometer which is particularly well suited for the knee, see Fig. 1. ${ }^{2}$ Thedata obtained with this system are then compared to the results of an optical motion capture system (Codamotion CX1, Charnwood dynamics, Leicester, UK).

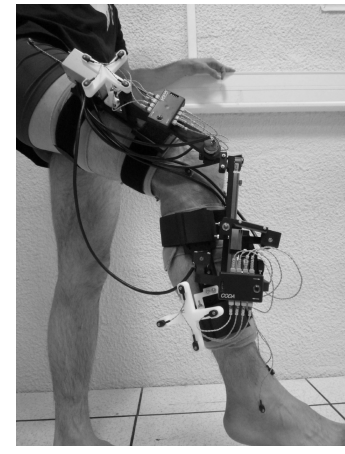

(a)

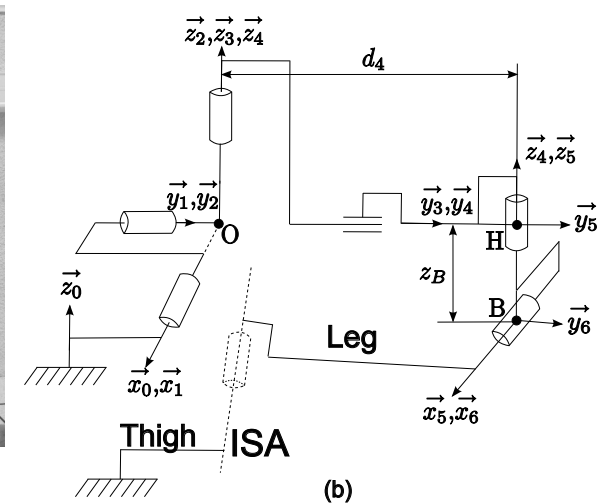

(b)

Fig. 1. (a) The six-axis goniometer and optical markers used in the experiments. (b) Frame assignment of the measurement system. The reference frame of the two systems is chosen to be $R_{0}=\left(O, \mathbf{x}_{\mathbf{0}}, \mathbf{y}_{\mathbf{0}}, \mathbf{z}_{\mathbf{0}}\right) . O$ is the point of intersection the first three axes.

\section{Methods}

For comparison, the ISA is determined by two different methods. The first, termed the "projection method", consists in the determination of the relative velocities of points belonging to the moving segment and projecting them onto the ISA. ${ }^{1}$ The second method, termed the "loop closure method", involves solving a linear system of equations obtained by closing the loop formed by the mechanism and the limb in order to determine the location of points located on the ISA, as well as their instantaneous velocities. 
Proceedings of the Thirteenth International Conference on Climbing and Walking Robots and the Support Technologies for Mobile Machines, CLAWAR 2010, in press.

\subsection{Projection Method}

The localization of the ISA is obtained from the computation of the orthogonal projections, $A_{i}^{\prime}$, of points $A_{i}$ attached to the moving segment onto the ISA. These projections are:

$$
A_{i} A_{i}^{\prime}=\left(\boldsymbol{v}_{A_{i}} \wedge \boldsymbol{w}\right) /\left(|\boldsymbol{w}|^{2}\right),
$$

where $\boldsymbol{w}$ and $\boldsymbol{v}_{A_{i}}$ are the angular and the translational velocities of the second segment at the point $A_{i}$. The relative translational velocity of the two body segments, which is also the instantaneous translational velocity of the anatomical joint can be estimated as follows:

$$
\boldsymbol{v}_{A_{i}^{\prime}}=\boldsymbol{v}_{A_{i}}-\boldsymbol{w} \wedge \boldsymbol{A}_{\boldsymbol{i}} \boldsymbol{A}_{\boldsymbol{i}}^{\prime}
$$

\subsection{Loop Closure Method}

By considering the subject's second limb and the anatomical joint as parts of the linkage, the loop-closure equation may be written

$$
\mathbf{J}_{P}(q) \dot{\boldsymbol{q}}-\left(\begin{array}{c}
\boldsymbol{w}_{06} \\
\boldsymbol{v}_{P}
\end{array}\right)=0
$$

where $\boldsymbol{q}$ is the vector of joint angles. $\mathbf{J}_{P}(q)$ is the Jacobian matrix written at a point, $P$, located on the ISA. The quantities $\left(\boldsymbol{w}_{06}, \boldsymbol{v}_{P}\right)^{\top}=$ $\left(\omega_{x}, \omega_{y}, \omega_{z}, v_{x}, v_{y}, v_{z}\right)^{\top}$ are components of the twist at $P$. The coordinates of the point $P$ and the values of $\left(\boldsymbol{w}_{06}, \boldsymbol{v}_{P}\right)^{\top}$ can be determined by solving the system of equations (3).

These two methods can be used when using a mechanical goniometer, whereas only the first is applicable to data from optical trackers.

\section{Results}

Our prototype goniometer has precision potentiometers to measure the joint angles that have a resolution of 0.02 degrees. Two experiments were conducted to evaluate performances. The angular calibration error was estimated at 5 degrees maximum. The sensor data were recorded at $1 \mathrm{kHz}$.

The motion capture system was used to measure the joint movements. The location data of the markers were recorded at $200 \mathrm{~Hz}$. The standard deviation of the position measurements of a static marker is $0.05 \mathrm{~mm}$ at $3 \mathrm{~m}$ on the $\mathrm{X}$ and $\mathrm{Z}$ axes and $0.3 \mathrm{~mm}$ on the $\mathrm{Y}$ axis.

In biomechanics applications markers are attached to the skin. Muscular contractions produce skin movements that introduce measurements errors 
Proceedings of the Thirteenth International Conference on Climbing and Walking Robots and the Support Technologies for Mobile Machines, CLAWAR 2010, in press.

that are difficult to quantify. In an effort to reduce this effect, a least-square optimisation method - that enforced the constraint of constant distance between markers - was applied on the raw kinematic data of the five active markers of each body segment.

\subsection{Test on a revolute joint}

The goniometer was fixed onto a revolute joint which was supposed to be perfect (see Fig. 2). The joint was moved at different speeds between 0 and 80 degrees.

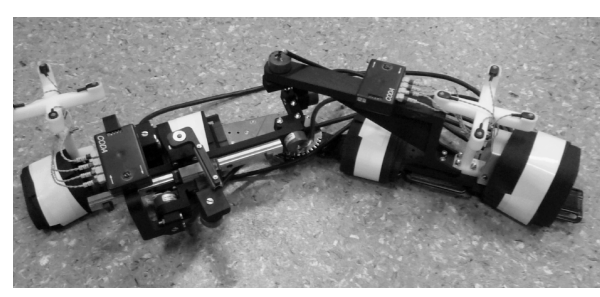

Fig. 2. The electro-goniomter and optical markers fixed onto a simple revolute joint.

Figures 3, 4, and 5 shows the result of this test. Due to the sensibility of the two methods for low angular velocities, data can not be computed when the absolute angular velocity is near to zero. Thus, for the test with the electro-goniometer, data are computed between the times $\Delta_{1}=$ $0.652 \mathrm{~s}$ and $\Delta_{2}=1.35 \mathrm{~s}$. For the test with the Codamotion system, data are computed between the times $\Delta_{3}=1.4 \mathrm{~s}, \Delta_{4}=2.42 \mathrm{~s}$ (see Figs. 4 and 5 ). The screw axis estimation computed by the electro-goniometer gives reliable values (see Fig. 5). The distance variation is less than $4 \mathrm{~mm}$ and the angular deviation is about 2 degrees during the whole motion.

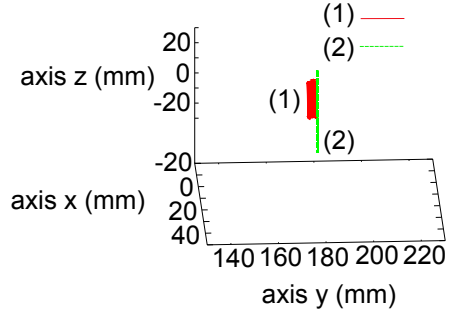

(a)

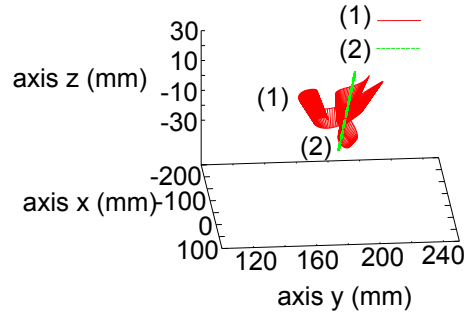

(b)

Fig. 3. A three dimensional view of the fixed revolute axis estimated by the goniometer. (a) Electro-goniometer result. (b) Codamotion result. (1) Estimated axis. (2) Real axis. 
Proceedings of the Thirteenth International Conference on Climbing and Walking Robots and the Support Technologies for Mobile Machines, CLAWAR 2010, in press.
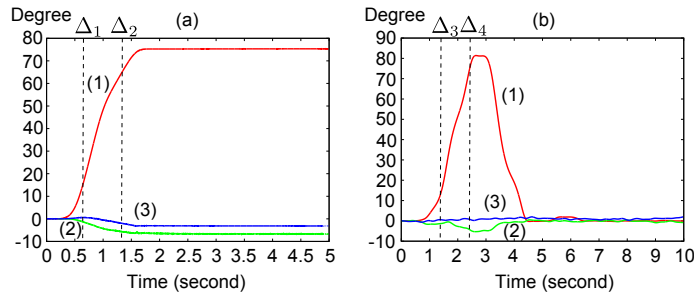

Fig. 4. Angular displacements: (a) Result of the electro-goniometer for a flexion movement, $\Delta_{1}=0.652 \mathrm{~s}, \Delta_{2}=1.35 \mathrm{~s}$. (b) Result of the Codamotion system for a flexionextension movement, $\Delta_{3}=1.4 \mathrm{~s}, \Delta_{4}=2.42 \mathrm{~s}$. (1) Angular displacement around x-axis. (2) Angular displacement around y-axis. (3) Angular displacement around z-axis.
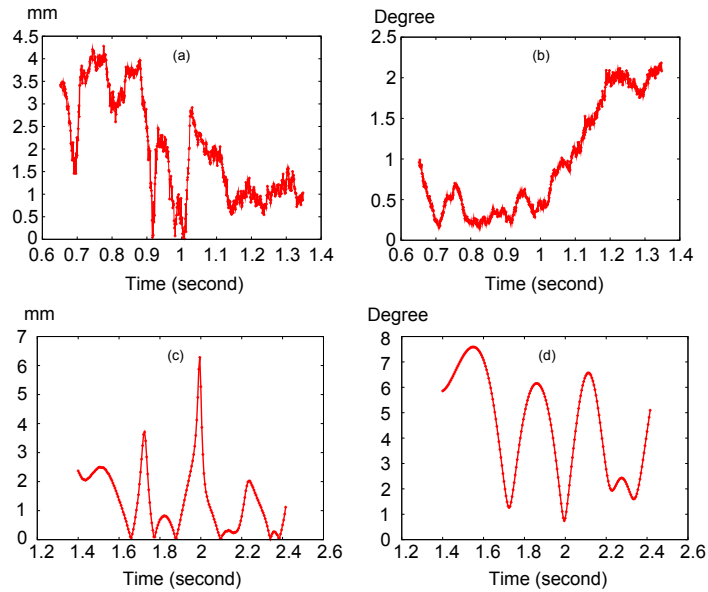

Fig. 5. The goniometer measurement results: (a) distance and (b) angular deviation of the estimated axis with regard to the real axis. The Codamotion measurement result: (c) distance and (d) angular deviation of the estimated axis with regard to the real axis.

\subsection{Test on a knee joint}

Other tests were carried out with the right knee of a subject to test the suitability of the goniometer. The first fixation plate was chosen to be the reference frame of measurement methods. Five active markers were fixed to the right lower leg. According to published recommendations, ${ }^{4}$ markers were placed on the lateral malleolus, medial malleolus, in the middle of the lateral and medial malleolus, the tuberosity of the tibia and the tibialis anterior. Other markers were fixed onto the second plate in order to check the reliability of the measurements. 
Proceedings of the Thirteenth International Conference on Climbing and Walking Robots and the Support Technologies for Mobile Machines, CLAWAR 2010, in press.
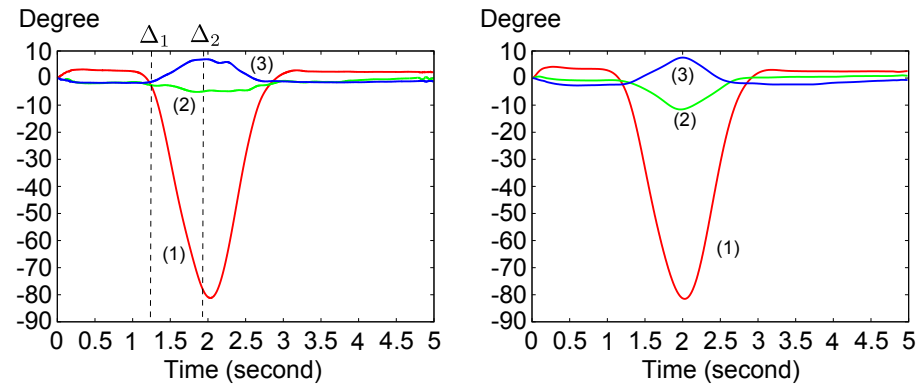

Fig. 6. Angular displacement estimation (a): by goniometer, (b): by Codamotion system. (1) Flexion - Extension. (2) Internal - External rotation. (3) Valgus - Varus. $\Delta_{1}=1.23 \mathrm{sec} ., \Delta_{2}=1.935 \mathrm{sec}$.
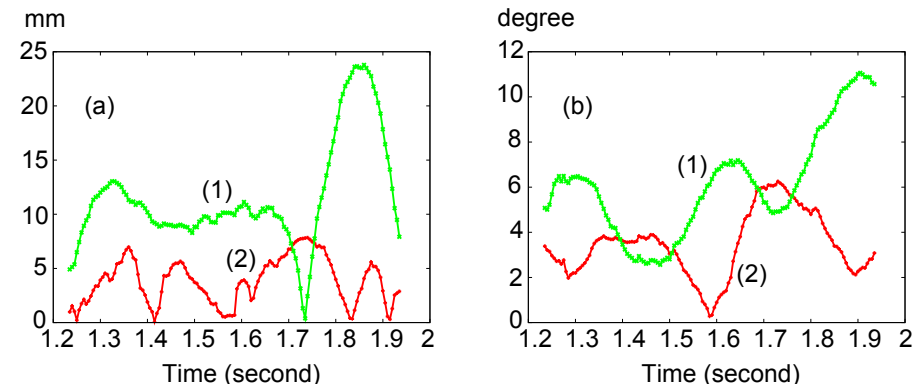

Fig. 7. (a) Distance and (b) angular deviation between the two instantaneous screw axes estimated by the two measurement systems. (1) Optical markers were placed onto the leg. (2) Optical markers were placed onto the second plate of the goniometer.

(a)

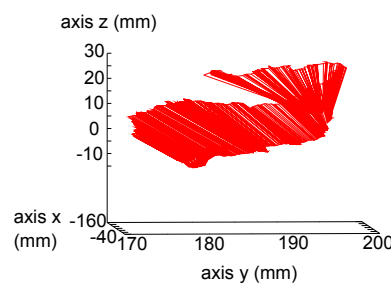

(b)

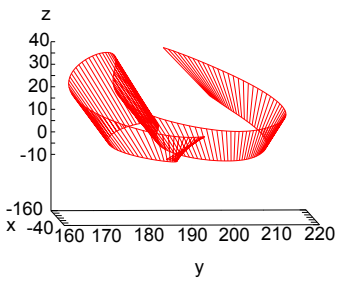

(c)

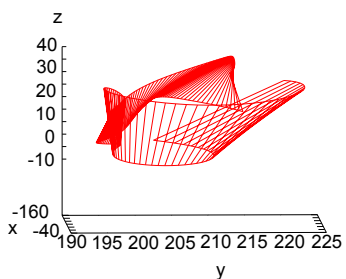

Fig. 8. The evolution of the ISA during the knee flexion. (a) ISA estimated by the electro-goniometer. (b) ISA estimated by the Codamotion system, with optical markers fixed onto the second plate of the goniometer. (c) ISA estimated by the Codamotion system, with optical markers fixed onto the leg. 


\subsection{Discussion}

The initial tests of the goniometer on a revolute joint provided coherent results. However, for the test on human knee, the estimated ISA and the angular values differed on the two systems, see Fig. 6 where a significant difference between the estimated angular values can be seen. Angular estimation errors can reach 10 degrees. On the other hand, we can see that the difference between the data obtained with the electro-goniometer and the Codamotion system vary when the second set of optical markers is fixed on the second plate of the goniometer and on the leg. This supports the notion that estimation is very sensitive to the displacement of the fixation plates and of the optical markers due to muscular contractions (see Fig. 7). The distance and the angular deviation between the two ISA can reach more than $20 \mathrm{~mm}$ and 10 degrees.

Angular estimation errors can be reduced by a precise calibration procedure, better sensors on the goniometer and by using more markers with the optical tracking device.

Nevertheless, the most likely source of errors is the numerical differentation needed to obtain velocity values. A number of approaches could be applied to improve numerical stability of the estimation algorithm, especially at low speeds.

\section{Conclusion}

A new method for the estimation of human anatomical joint motions, using a multi-linkage goniometer was proposed in this paper. Simulations and experimental tests were carried in order to evaluate the performance of this measurement system. Simple tests on elementary joints, such as revolute joints, provided reliable results. Tests on the human knee joint, showed significant differences between measurements provided by the electro-goniometer and a motion capture system using optical markers. In future work, efforts will be directed at improving the reliability of the estimation of the ISA with the two approaches. 
Proceedings of the Thirteenth International Conference on Climbing and Walking Robots and the Support Technologies for Mobile Machines, CLAWAR 2010, in press.

\section{References}

1. B. Bru and V. Pasqui, A new method for determining the location of the instantaneous axis of rotation during human movements, (Computer Methods in Biomechanics and Medical Engineering, Vol. 12, sup. 1, pages 65-67, 2009).

2. V.A.D. Cai, P. Bidaud, V. Hayward and F. Gosselin Design of Self-Adjusting Orthoses For Rehabilitation, (Proceedings of the 14th IASTED International Conference Robotics and Applications (RA 2009), paper n. 664-030, pages 215-223, 2009).

3. R.M. Ehrig, W.R. Taylor, G.N. Duda, and M.O. Heller, A survey of formal methods for determining the centre of rotation of ball joints, ( $\mathrm{J}$ Biomech 39 (2006): 2798-2809).

4. S. Van Sint Jan, Color Atlas of Skeletal Landmark Definitions, (Churchill Livingstone, Elsevier,Philadelphia, 2007).

5. G.L. Kinzel, A.S. Hall and B.M. Hillberry, Measurement of the total motion between two body segments - I. Analytical development, (J. Biomechanics, Vol. 5 , pages $93-105,1972)$.

6. T. Monnet, E. Desailly, M. Begon, C. Valle, and P. Lacouture, Comparison of the SoRE and HA methods for locating in vivo the glenohumeral joint centre, (J Biomech 40 (2007): 3487-3492).

7. M.A. Townsend, M. Izak and R.W. Jackson, Total motion knee goniometry, (J. Biomechanics, Vol. 10, pages 183-193, 1977).

8. H.J. Woltring, R. Huiskes, A. de Lange, and F.E. Veldpaus, Finite centroid and helical axis estimation from noisy landmark measurements in the study of human joint kinematics, (J Biomech, 18(5):379-389, 1985).

9. G. Wu, F.C.T. van der Helm, H.E.J. DirkJan Veeger, M. Makhsous, P. Van Roy, C. Anglin, J. Nagels, A.R. Karduna, K. McQuade, X. Wang, F.W. Werner, B. Buchholz, and International Society of Biomechanics, Isb recommendation on definitions of joint coordinate systems of various joints for the reporting of human joint motionpart ii: shoulder, elbow, wrist and hand, (J. Biomechanics, 38(5):981-992, 2005). 